\title{
Using timetabling optimization prototype tools in new ways to support decision making
}

\author{
M. Forsgren ${ }^{1}$, M. Aronsson ${ }^{1}$, S. Gestrelius ${ }^{1} \&$ H. Dahlberg ${ }^{2}$ \\ ${ }^{1}$ SICS, Swedish Institute of Computer Science, Sweden \\ ${ }^{2}$ Trafikverket, Sweden
}

\begin{abstract}
The Swedish infrastructure manager Trafikverket is funding research for timetabling optimization tools as part of their overall mission to utilize the existing infrastructure more efficiently. Currently, Trafikverket is modernizing both planning processes and the IT architecture, and will soon be ready to start using optimization tools on a broad scale. Meanwhile, innovative uses of a prototype developed at SICS has shown how a prototype does not necessarily merely serve to pave the way for a future, large-scale implementation. This paper shows how computers in railway planning, coupled with OR techniques, relevant data and apt modeling, can help provide a future user with valuable insights even before the fully-fledged tool is in place.

Keywords: timetabling, optimization, prototype tool, planning, robustness.
\end{abstract}

\section{Introduction}

The quality of the railway timetable ultimately affects the transportation market share held by railroads. Research in the field indicates that optimization has the potential of increasing the timetable quality substantially, with the Dutch timetable being one of the most comprehensive and illustrative examples to date [1].

Introducing optimization tools in an organization unfortunately does not only involve designing and installing the relevant software. The Swedish experience is that the processes and the IT architecture of the infrastructure manager need to be updated in order to fully benefit from optimization tools. This is probably true for most countries where timetabling traditionally has been essentially a manual task.

In Sweden, a prototype tool for timetabling called Marackasen has been developed by researchers at SICS (Swedish Institute of Computer Science) for 
Trafikverket (the Swedish Transport Administration). However, due to the reasons outlined above, a full implementation of the tool will take time.

The research at SICS is not limited to the tool but also aims to modernize processes. Marackasen has gradually established itself as a tool supporting the ongoing research and demonstrating the potential of suggested measures. This paper describes real instances of innovative uses of the prototype, illustrating that a prototype can be very useful in its own right.

The paper is organized as follows. First a brief introduction to the tool is given, to provide a background. Then four real cases are presented, demonstrating how the tool can be used in addition to facilitating the timetable planners in their daily work. Last a concise summary of the usefulness of the tool is given.

\section{Brief overview of Marackasen}

This section gives a very brief overview of the tool that was previously described in "The Maraca: a tool for minimizing resource conflicts in a non-periodic railway timetable" [2]. It is a prototype developed by SICS for Trafikverket for optimization of timetables.

A non-exhaustive list of timetable properties that can be optimized with Marackasen are: running times, the deviation from the input timetable, and the total time the trains are waiting for other trains to pass at meeting locations. Parameters stating whether the original meeting locations can be changed or not, and if any event times are pre-defined, also highly affect the solution.

Marackasen is written in SICStus Prolog and Java. Marackasen parses and transforms timetabling data into a linear optimization problem and outputs it to a file that is fed to IBM ILOG CPLEX Optimizer. The solution from CPLEX is read by Marackasen and visualized in a GUI.

Timetabling data suitable as input to Marackasen can be exported as XML from TrainPlan, the timetabling tool used at Trafikverket. TrainPlan, a commercial railway planning tool offered by Funkwerk IT, is mainly a visualization tool and database. It manages huge amounts of data and produces distance-time graphs, but it does not offer any optimization capabilities.

Marackasen deliberately uses the same network abstraction as TrainPlan. The network is divided into nodes and links connecting the nodes. The nodes are stations, selected block signals and points. These are seen as resources that can host a specified number of trains simultaneously.

The timetabling rules that have been stipulated by Trafikverket [4] are implemented in Marackasen and include requirements such as least time separation between trains at stations depending on the station type and the stopping behaviors of the trains. On single track, no two trains may be on the same link at the same time. For double track, trains in the same direction need to respect a specified headway. 


\subsection{Using Marackasen}

The input to Marackasen is a draft timetable. It is used as the baseline for finding a better solution. The motivation for using this approach is that it resembles the way the timetable planners work today, while additionally allowing the timetable planner to make larger, strategic moves manually, leaving the details of conflict resolution and optimization to the tool.

Note that, when using Marackasen for conflict resolution, finding a conflict free timetable is equivalent to minimizing the number of resource conflicts and finding a solution that contains no such conflicts.

\section{Proving a concept}

This section explains how Marackasen was used to show that a new planning approach referred to as Successiv tilldelning, or Successive Allocation, allows for more efficient use of the infrastructure than the current approach.

Whereas the basic principles governing Successiv tilldelning are easy to explain, explaining in general terms the practical consequences and the benefits of adopting the concept is harder, since it is not possible to quantify the effects in a simple way that catches them all.

To understand the significance of the contribution of Marackasen in this context, a brief overview of the concept and the ideas behind the concept are presented.

\subsection{The current planning practice}

Railway traffic in Sweden can in general terms be described as mixed and nonperiodic, and a large part of the railway infrastructure consists of single track lines. The railway is completely deregulated.

The train plan for the upcoming year is officially published in the middle of September each year. During the ad hoc process that follows, existing train paths may be canceled and new train paths added. New train paths are only allowed to use remaining capacity. Modifying a train path to give room to a new one, or for any other reason, is not allowed.

In practice, train paths are modified on a day-to-day basis during operations, as the dispatchers naturally do not follow the yearly plan slavishly. Not updating the plans, but instead keeping train paths the way they were originally planned even when they will clearly not be dispatched that way, is obviously not practical from any point of view. It forces the dispatchers to improvise more than would otherwise be the case, and it is likely that the result is suboptimal.

\subsection{Successiv tilldelning}

Successiv tilldelning is currently being implemented at Trafikverket [4]. It is a concept based on ideas developed at SICS, mainly as a reaction to the significant mismatch between the train plan and the actual operations. The main reason for 
this mismatch was identified as the stilted process after the yearly train plan is published, dictating that there must not be any changes made to the train paths in the plan.

Given the general properties of railway traffic in Sweden, and specifically its non-periodic nature, researchers at SICS saw the potential in allowing the plan to be updated continuously. In addition, by making the yearly train plan slightly less rigid, even more freedom to adjust to each individual day is obtained. This can be done, SICS argued, in a safe way that preserves all the important properties of the train services.

The basic idea for the new approach is that existing train paths may be modified as long as no important arrivals and departures are affected. These arrivals and departures are events mainly of commercial nature, but any kinds of events of significance to the RU are of course possible. It is assumed that Trafikverket and the RU:s define, in contracts, which arrivals and departures that should be respected at all times. The locations for the trains where such agreements exist are called delivery commitment locations.

Using Successiv tilldelning frees up infrastructure capacity. The extra capacity can be used for making the plan more robust and more resilient to knock-on delays, or for adding more train paths to the timetable.

\subsection{Adding a train to an existing timetable}

Intuitively, a less rigid timetable along the lines of Successiv tilldelning should make more room for new train paths in a way that should be fairly easy to demonstrate. The opportunity to test this on a real case arose in the fall of 2009 when one of the RU:s for passenger trains in Sweden asked Trafikverket for a new train path during the ad hoc process.

The RU wanted to see if it was possible to compete with the bus traffic between two cities in the northern part of the country. To illustrate the usefulness of the suggested new approach, SICS used Marackasen to show what the train path would look like using Successiv tilldelning compared with using the current approach.

\subsubsection{Using the current approach}

The original application involved a departure at 6.14 and an arrival at 9.40 . The best obtainable running time for the train, ignoring other scheduled trains, was 2 hours and 48 minutes. Since the RU had tried to take into account existing train paths, they applied for a more realistic running time of 3 hours and 26 minutes.

Closer examination showed that the earliest a train of this type could arrive at the end destination was at 10.22 if it was to depart at around 6.14 and if it was added to the train plan using the current approach where no existing train paths can be changed in any way.

Figure 1 shows what such a train path would have looked like. Minimizing the running time, the departure would have been 6.30 instead of 6.14 , and the running time 3 hours and 52 minutes, 1 hour and 4 minutes longer than the fastest possible running time for the train type in question, and 26 minutes longer than the RU was 


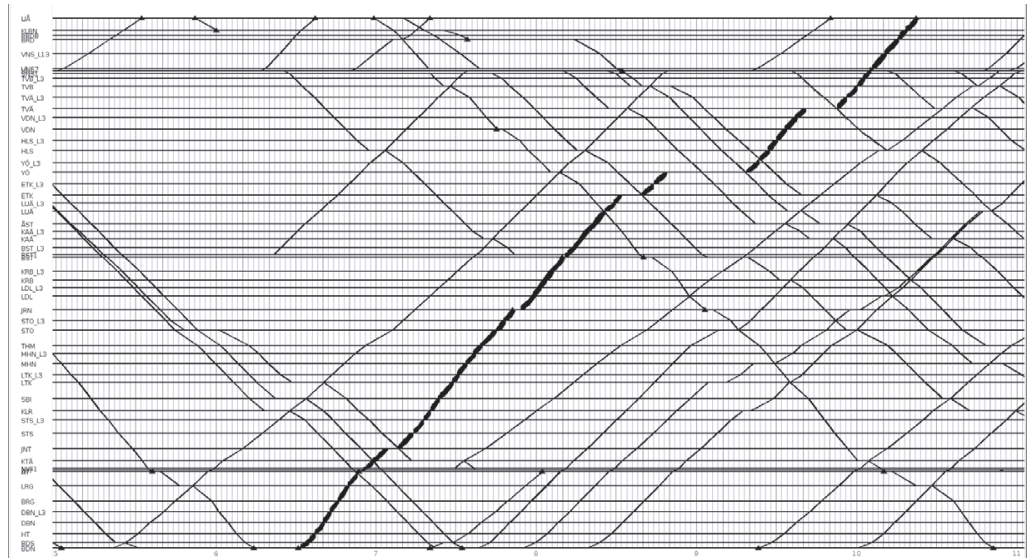

Figure 1: The train path (fat line) when using the current approach.

hoping for. The arrival at the final destination would have been 42 minutes later than the one proposed in the application, due to the delayed departure.

\subsubsection{Using the new approach}

Focusing instead on respecting arrivals and departures at the delivery commitment locations, Figure 2 shows what the train path would have looked like using Successiv tilldelning.

Successiv tilldelning would allow for a running time of 3 hours and 7 minutes which is 45 minutes shorter than with the current approach. The key to this is a slight modification to the surrounding trains, without any of their important arrivals or departures suffering in any way. The train was meant to run 80 times, totaling a saving of 60 hours in running time compared with the current approach. Not to

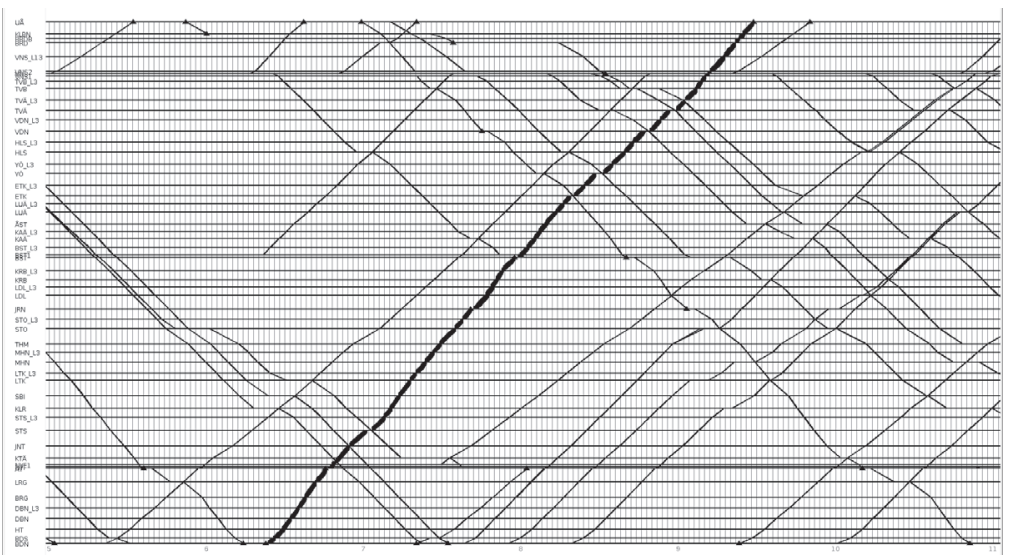

Figure 2: The resulting train path (fat line) after using the new approach. 
mention the fact that this short running time could seriously compete with other transportation modes.

\subsection{Conclusions}

While a train could have been added to the timetable using either approach, using the current approach, the train would have got a train path with a long running time, and it would probably not have been able to compete with the bus. The Successiv tilldelning approach gives the train a far better train path, without compromising any important arrivals or departures.

What is made clear by this example is that the capacity for a decent train path indeed existed, but was hidden by the inflexibility of the current approach. The train path finally awarded to the RU was similar to the one constructed using Successiv tilldelning. Reports from Trafikverket showed that the punctuality in the area during the time for this train did not decrease, indicating that the train path in question was definitely not only a theoretical construct.

It would have been possible, although much more time-consuming, to demonstrate Successiv tilldelning manually in this particular case. However, whereas it is difficult to prove that a manual solution is correct and the best possible one, the results from Marackasen could be trusted to be correct and optimal, making the case even stronger.

\section{Demonstrating potential}

Encouraged by the initial tests that illustrated the advantages of using Successiv tilldelning when adding individual trains to an existing timetable, the decision was made to perform more systematic calculations with Marackasen to explore the general potential.

Modifying and extending the previous test to incorporate existing trains in the train plan rather than trying to add more trains, the aim was to see how much running time in total it was possible to save with Successiv tilldelning relative the current approach. The calculations and their results are outlined in this section. The optimization was carried out on three randomly selected days for a part of the train plan of 2010 of reasonable size.

\subsection{Running time components}

The running time of a train consists of several components, some of which are not possible to reduce in length. Slack is here defined as the extra time given to a train relative the minimum time it needs, including any planned stops, to cover the distance of the whole trip.

Slack that is typically used to facilitate for trains to meet or overtake is sometimes referred to as pathing time, to separate it from time supplements that are added to compensate for varying driver behaviors and other natural variations, including buffer time against small, everyday perturbations. 


\subsection{The minimization}

For the minimization of the running time total of all trains, it was assumed that the only running time component that could be changed was the time explicitly declared as pathing time. Stop types for the trains indicating commercial activities defined the delivery commitment locations for the trains, and at such locations, trains were not allowed to arrive later than the time given in the timetable, nor depart earlier than the given departure time.

Last but not least, since it could not generally be assumed that all locations were equally suitable for all train meetings, the locations for the train meetings in the timetable were fixed during the minimization.

Table 1: Minimizing the running time total.

\begin{tabular}{|c|r|r||r|r||c|}
\hline \multicolumn{7}{|c|}{ Running time [s] } & \multicolumn{3}{c|}{ Difference } \\
Scenario & original & optimized & \# seconds & \multicolumn{1}{c|}{$\%$} & CPU time [s] \\
\hline \hline A & 179992 & 155509 & -24483 & -13.6 & 0.23 \\
B & 277728 & 248196 & -29532 & -10.6 & 0.79 \\
C & 280420 & 246004 & -34416 & -12.3 & 1.65 \\
\hline \hline D & 89218787 & 85347726 & -3871061 & -4.3 & 3.71 \\
\hline
\end{tabular}

Table 1 summarizes the results of the optimizations for the three days, Scenario A-C, and the yearly plan, Scenario D. The reduction in running time total is in the same order of magnitude for all three individual days, indicating that this is a general property of the plan. The optimization by CPLEX 12.1.0 took less than two seconds when optimizing on a single day, and a little less than four seconds for the yearly plan, when run on a ThinkPad T60, with Intel processor Core(TM)2 Duo, $2 \mathrm{GHz}$, under Ubuntu Linux.

The calculation on the yearly plan was performed to be able to distinguish between the contribution of the optimization of running times in general on the one hand, and the actual contribution of Successiv tilldelning on the other. Subtracting the savings in running time of $4 \%$ that Marackasen in this way showed was possible to reduce in the yearly plan, the rest, $6-9 \%$, could in fairness be attributed to the new planning approach.

\subsection{Conclusions}

The results of the calculations described in this section worked as an eye-opener at Trafikverket. The experiment proved that the current planning approach hides capacity in a way that is possible to quantify. Coupled with a powerful optimization tool, Successiv tilldelning would indisputably provide a way of uncovering truly non value adding components of the running times. 
Studying the implications of the results, the main contribution of uncovering capacity in this way is that it makes timetable planners and dispatchers aware of the daily variations in a whole new way, thereby enabling them to make the best possible use of existing buffers on a day-to-day basis.

\section{Verifying a model}

In 2007, The Swedish Rail Administration (Banverket) was officially ordered by the Swedish Rail Agency (Järnvägsstyrelsen) to implement priority criteria to be ready for use in December 2009 [5]. The order was based on EU Directive 2001/14/EC that states that the infrastructure manager may employ priority criteria to allocate infrastructure capacity when the infrastructure has been declared congested [6]. Moreover, quoting the Directive, "The priority criteria shall take account of the importance of a service to society, relative to any other service which will consequently be excluded."

In relatively short time, Banverket thus needed to find a way of comparing the socio-economic costs between different possible timetables. A project to this end was initiated, and SICS were asked to participate as consultants.

\subsection{The priority criteria model}

The basic assumption for the model is that the original applications reflect what would have been the best solution from a socio-economic point of view, had it been possible to schedule all trains without modification. Any deviation from the original application increases the cost. Also, trains are given priority categories, representing their relative value to society.

The following are some, but not all, of the changes to the original applications that affect the cost of a solution: change of departure time, increased running time, violation of time windows, increased route length, broken associations, and rejected trains. For further details, the interested reader is referred to [7] and the Swedish Network Statement [8].

\subsection{Minimizing the socio-economic cost with Marackasen}

During the project, SICS identified the potential of using Marackasen to minimize the cost of the timetable, thus showing what the timetable would look like according to the stipulated priority criteria, if they were in force. If the resulting timetable would be counter-intuitive to the project group, the model might have problems, SICS argued.

A main example of limited size was used to test the reasoning during the project. Even so, actually minimizing the cost manually would have been too time-consuming for the project group. Despite the involved definition of the cost function, using Marackasen on the main example showed to be possible from a computational point of view thanks to the linear property of the model. 
The data that was used for the minimization represented a week of traffic. The timetable after minimizing the cost was compared with the manual solution constructed by one of the timetable planners at Banverket. As expected, the cost of the timetable solution was lower for the optimized one compared with the human solution. In figures, the solutions of the timetable planner and that of Marackasen was 95 hours compared to 88 for the total running time of the trains, and 5 hours and 41 minutes in total for the changes in departure times compared with 3 hours and 45 minutes for the solution produced by Marackasen.

\subsection{Conclusions}

The main purpose of the model is to provide a transparent and predictable way of resolving conflicts. It is not a perfect model, but still serves its purpose since it can be used to compare solutions, especially when the difference in cost is significant, Trafikverket can argue that the one with the lowest cost is the better one from the point of view of society.

There were significant differences between the man-made and the optimized solutions. After closer examination, this indicated more than just the expected difference due to the imperfection of manually performed timetable construction versus the power of the computer. There was indeed a flaw in the model that had to do with how trains of certain categories were valued. Although the mistake was not identified primarily because of the test with Marackasen, the test gave especially the timetable planner an early indication that something was not quite right in the model.

\section{Illustrating problem characteristics}

A timetabling tool with optimization capabilities obviously facilitates playing with different ideas on what to optimize since it is relatively easy and quick to test the ideas and see their consequences. In the case of examining robustness properties and how to define robustness, Marackasen and its GUI also definitely helped in shaping an idea.

With Successiv tilldelning, as described in Section 3.2, a deviation from the plan is not regarded as a problem unless it causes trains to arrive late at pre-defined, strategic locations; if a train does not arrive late at such a location, it is not regarded as being delayed with respect to the RU. An aspect of this relating to robustness involves determining to what extent trains in a timetable are free to utilize the capacity before they actually start delaying other trains according to the definition just mentioned. This property may be something that one would want to maximize to achieve a more robust timetable.

The idea of redistributing slack geographically by changing locations for the train meetings and train overtakings during operations was recently published by SICS, and for the details the interested reader is referred to the full paper [9]. Here the basics of the idea are outlined so that it is possible to understand how Marackasen was used in shaping the idea. 


\subsection{Maximizing delivery robustness of train timetables}

When trains are scheduled with the Successiv tilldelning approach, only a subset of arrival and departure times are published in the timetable, namely the arrivals and departures at the delivery commitment locations (see Section 3.2). This gives a varying degree of flexibility to arrivals and departures at the remaining locations.

Using the definition of slack given in Section 4.1, the slack between two delivery commitment locations is the sum of all extra time for the train between those two locations. In Successiv tilldelning, the size of the slack is mainly determined by the number of scheduled meetings for the train between the two locations, but the exact locations where the slack will be used during operations need not be fixed. The point of Successiv tilldelning is to leave as much freedom as possible for as long as possible during the planning process.

The effects of slack can be described as if every arrival and departure has a continuous domain of possible values. Combined with the characteristics of the vehicle, it is possible to show which area in a distance-time graph that a train in a timetable is confined to. Such an area is here referred to as a train corridor.

Studying such displayed train corridors in combination in Marackasen, it became clear that overlapping corridors effectively confine the possible locations for meetings (see Figure 3). This, in combination with the knowledge that changing meeting locations can redistribute slack, lead to the conclusion that there is an important relation between the number of possible meeting locations in the overlapping corridors and robustness during operations.

Trafikverket agreed that this idea should be exploited and heuristics were developed. Finally a paper was submitted to a conference and published.
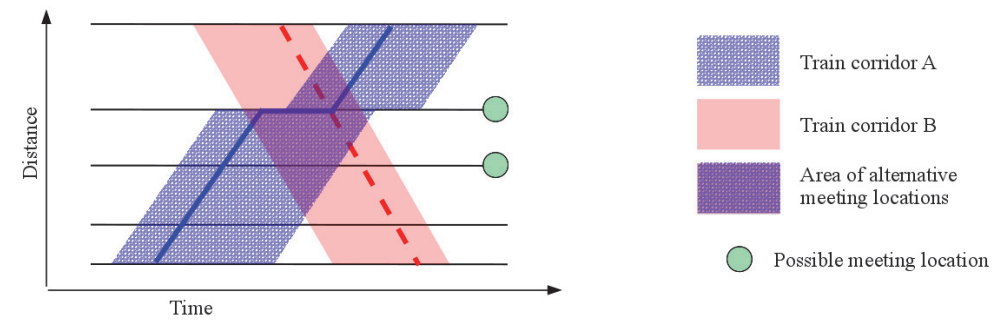

Figure 3: Overlapping train corridors confining possible meeting locations.

\subsection{Conclusions}

Maximizing the delivery robustness along the lines given here, without compromising other timetable qualities, is clearly a sound approach. In the future, 
this idea might very well affect the way different timetable solutions are analyzed and weighed against each other at Trafikverket.

Also, displaying train corridors has increased the understanding of the problem characteristics at SICS and at Trafikverket. For instance, any train path that has only one possible realization will be displayed as a fine line, thus highlighting trains that must not be changed in any way during operations. The likelihood that such a train will be on time is in practice very small, and such train paths might be undesirable in the first place.

\section{Summary}

The prototype optimization tool Marackasen has been used to show the potential in several concepts, for example a new planning approach called Successiv tilldelning.

Using Marackasen to investigate how a train path could be added to an existing train plan, the difference between using the current approach and Successiv tilldelning could be demonstrated. In the particular case, the difference was substantial and clearly illustrated the power of the concept.

Minimizing the overall running times in a train plan with Marackasen, using Successiv tilldelning, showed the potential of this new approach on a broader scale and complemented the specific case of the isolated improved, train path. Together, the two cases comprised an even stronger case for Successiv tilldelning and also indicated the order of magnitude of capacity that is currently hidden and might be uncovered.

In another project, Marackasen helped project members to reflect on the choices made for a model measuring the socio-economic value of a timetable. Last, but not least, using the prototype has shown to evoke ideas that otherwise would not have been thought of, significantly contributing to a deeper understanding of the problems the infrastructure manager is facing.

\section{Acknowledgement}

The research presented in this paper was funded by Banverket (2005-2010) and Trafikverket (2010-) via the projects Den Dynamiska TågPlanen, grant number S04-3104/AL50, Tågplan 2015 (F08-6472/AL50) and Marackasen (F086473/AL50).

\section{References}

[1] Kroon, L., Huisman, D., Abbink, E., Fioole, P.J., Fischetti, M., Maroti, G., Schrijver, A., Steenbeek, A. and Ybema, R., The new Dutch timetable: The OR revolution. Econometric Institute Report EI 2008-19, Erasmus University Rotterdam, Econometric Institute, 2008. 
[2] Forsgren, M., Aronsson, M., Kreuger, P. and Dahlberg, H., The Maraca: a tool for minimizing resource conflicts in a non-periodic railway timetable. Proceedings of the The 4th International Seminar on Railway Operations Modelling and Analysis, Rome, Italy, 2011.

[3] Banverket, Riktlinjer för tidtabellskonstruktion på statens spåranläggningar. Regulatory document TF601 published by the Swedish Rail Administration, 2000.

[4] Trafikverket, Aktuella projekt - successiv tilldelning. Project web page, 2012. http://www.trafikverket.se/Foretag/ Trafikera-och-transportera/Trafikera-jarnvag/ Successiv-tilldelning/.

[5] Järnvägsstyrelsen, Föreläggande rörande Banverkets järnvägsnätsbeskrivning. Document 2006-1473/22, the Swedish Rail Agency, 2007.

[6] Directive 2001/14/EC of the European Parliament and of the Council of 26 February 2001 on the allocation of railway infrastructure capacity and the levying of charges for the use of railway infrastructure.

[7] Graylay, C. and Franzén, T., Priority criteria for timetable planning - capacity allocation for maximum social economic benefit. Railway Strategies, web magazine, 2011. http://www.railwaystrategies.co. uk/article-page.php?contentid=12413\&issueid=374.

[8] Trafikverket, Järnvägsnätsbeskrivning 2012, appendix 4.2. Network Statement published on the web, 2012. http://www.trafikverket.se/ PageFiles/40861/bilaga_4_2_prioriteringskriterier_jnb_ 2012_120402.pdf.

[9] Gestrelius, S., Aronsson, M., Forsgren, M. and Dahlberg, H., On the delivery robustness of train timetables with respect to production replanning possibilities. Proceedings of the The 2nd International Conference on Road and Rail Infrastructure (CETRA), Dubrovnik, Croatia, 2012. 